\title{
EL MARTIRIO DE LOS DIEZ MIL. DANTE EN LA PINTURA DE VITTORE CARPACCIO
}

\author{
THE MARTYRDOM OF THE TEN THOUSAND. DANTE IN THE PAINTING \\ BY VITTORE CARPACCIO
}

Jesús María González de Zárate

Universidad del País Vasco

ABSTRACT: Vittore Carpaccio, around 1515, offered us the visual chronicle of the torture suffered by the ten thousand martyrs on Mount Ararat that had been previously related in Carolingian martyrologies. In some, such as that by Usuard, printed in Venice by the date of the painting, we can find the literry source of its composition. The tale focuses on the battle, the victory, and the martyrdom to which the soldiers were condemned, as well as on the divine promise of resurrection. This latter aspect is not found in other depictions of the theme, and Carpaccio offers it to us following the same cosmic architecture that Dante proposes in his Comedia.

KEYWORDS: Carpaccio, Martyrs of Mount Ararat, Usuard, Martyrology, Dante, Iconography.

RESUMEN: Vittore Carpaccio, hacia 1515, nos ofrece la crónica visual del suplicio de los diez mil mártires en el monte Ararat que ya quedó relatada en martirologios carolingios. En alguno, como el de Usuardo, impreso en Venecia para esta fecha podemos encontrar la fuente literaria de la composición. El relato repara en la batalla, en la victoria y en el martirio al que fueron condenados, también en la divina promesa de la resurrección, aspecto este último que no encontramos en otras representaciones del tema, y que Carpaccio nos ofrece siguiendo la arquitectura cósmica que propone Dante en su Comedia.

PALABRAS CLAVES: Carpaccio, mártires del monte Ararat, Usuardo, martirologio, Dante, Iconografía. 
Poco se conoce sobre la biografía de Vittore Carpaccio (ca.1465-1526). Esencialmente fue un artista pintor afincado en Venecia en el que domina el tratamiento del paisaje urbano a través de una visión personalista e imaginativa. La luz y el color, así como el gusto por el detalle, definen sus composiciones en base a la influencia de Gentile Bellini en el tratamiento de los espacios abiertos y de Antonello da Messina en el sentido del realismo flamenco por cuanto, el siciliano, afincado en Venecia, fue su introductor. Muchas de sus obras nos ofrecen una iconografía sacra en base a grandes lienzos que nutren toda una serie de secuencias que, en la actualidad, fuera de su contexto unitario, se dan cita en diferentes museos del mundo.

Las fuentes literarias consultadas por el artista son plurales. No falta la conocida y divulgada para la fecha Leyenda Dorada de Vorágine, así como el Martirologio Romano como tendremos ocasión se comprobar seguidamente. Los Tratados de mitología no están ausentes, como se observa en los trabajos de Hércules que dispone en la Presentación de María (1504-8. Pinacoteca de Brera. Milán). La poesía de Dante, entre otras fuentes, también ocupa las creaciones de Carpaccio.

El presente estudio se centrará en el argumento sobre Los diez mil mártires de Ararat, poniendo de relieve las fuentes iconográficas que se dan cita en su representación.

\section{LOS DIEZ MIL MÁRTIRES DEL MONTE ARARAT}

El 22 de junio es la fecha en el Martirologio Romano para conmemorar la fiesta de estos martirios que transcurrieron bajo los emperadores Adriano y Antonino en el siglo II. Los mártires responden a los soldados que siguieron en su fe a Acacio de Armenia, posteriormente san Acacio, y que sufrieron todo tipo de tormentos hasta su muerte.

El Martirologio Romano fue encomendado, ante el desorden que existía sobre biografías de mártires cristianos, por el Papa Gregorio XIII al cardenal César Baronio y fue editado en Roma en el año 1583 con el título: Martyrologium Romanum ad novam Kalendarii rationem et ecclesiasticae histórice veritatem restitutum, Gregorii XIII Pont. Max. iussu editum. En la relación de mártires se da cuenta de los sucesos en el monte Ararat. La fuente de Baronio se fundamenta en anteriores tratados como el del siglo VIII escrito por el benedictino Beda el Venerable $\mathrm{y}$, esencialmente, en el martirologio de Usuardo, también monje benedictino de esa misma centuria, que gozó de gran difusión en la Europa medieval y por ello en nada extraña que fuera impreso en la Venecia en el año 1498 y se convirtiera en fuente para nuestro artista.

La Leyenda da cuenta de la sublevación de algunos pueblos en torno al Éufrates contra el Imperio. Miles de soldados romanos huyeron de la contienda ante el gran número de enemigos; otros, unos nueve mil, buscaron la muerte en el enfrentamiento. La súplica a los tradicionales dioses paganos tan solo generaba desgracias en las huestes romanas. En estas circunstancias, un ángel les anunció la victoria de pedirse auxilio a Cristo, verdadero Dios. Finalmente convertidos a la fe cristiana, obtuvieron su recompensa. Los soldados, tras la victoria, propagaron sus nuevas creencias de fe y por ello recibieron la amenaza del castigo. En estas circunstancias los trasladaron al monte Ararat donde otra vez fueron amonestados por ambos emperadores para que retornasen al tradicional culto pagano. Ninguno de los legionarios, en voz de su comandante Acacio, hizo apostasía de su fe y por ello fueron condenados a muerte. Así, en primera instancia fueron lapidados, pero el martirio no tuvo su efecto ya que las piedras se volvían hacia quien las lanzaba, e incluso secaba las ma- 
nos del tirador. En este momento, mil soldados más observaron el milagro y no dudaron en abrazar la nueva fe. Con ello fueron diez mil los mártires. A todos los desnudaron y flagelaron, también tuvieron que andar por caminos espinosos donde gracias a los ángeles se apartaban las púas; fueron coronados de espinas y se les atravesó el costado con lanzas, recordando de esta manera el martirio de Cristo. El horror finalizó con la crucifixión de un importante número en el monte Ararat mientras otros eran arrojados por los peñascos para caer sobre estacas. Tras tan horrenda muerte los ángeles recogieron sus cadáveres para cumplir la solicitud hecha a Dios de que todos ellos volvieran a la vida en cuerpo y alma. En varios aspectos, la narración recuerda el tema de san Mauricio y la legión tebana como lo podemos observar en la pintura de Pontorno.

El relato, en toda su extensión, queda recogido en la pintura de Carpaccio quien, de manera erudita y novedosa, sujeto a las fuentes como veremos, expresa el cumplimiento de aquella promesa, en consecuencia la llegada al cielo. Curiosamente, como veremos, la fuente literaria para traducir visualmente la resurrección de los mártires, se presenta siguiendo la figura de Dante.

\section{EL ARGUMENTO HAGIOGRÁ- FICO LLEVADO A LAS ARTES}

El tema de los diez mil mártires en el monte Ararat aparece con cierta profusión en Venecia, la explicación bien se puede encontrar en el peligro turco que atentaba y hacía peligrar sus intereses comerciales en el Mediterráneo oriental. Alberto Durero, tras su viaje a la ciudad del Adriático entre los años 1494-95, representó este tema siendo uno de los pioneros en la creación del tipo iconográfico. En su entalladura (1496, Viena, Albertina, die Grafische sammlung), el gra- bador nos presenta en la zona inferior dos aspectos: por un lado la autoridad, con corona y turbante, decidiendo los castigos; por otro, el suplicio y tortura a un mitrado. La zona superior queda ocupada por los diferentes castigos a los soldados. Así, semidesnudos, son arrojados al abismo, flagelados y ajusticiados por la espada.

Doce años después, en 1508, Durero lleva el tema a la pintura. El encargo proviene de Federico el Sabio para la cámara de las reliquias en su paladio de Wittenberg donde se custodiaban algunos restos de los citados mártires (Viena, Kunsthistoriches Museum) [fig. 1]. En este caso el tipo iconográfico se centra en las crucifixiones. Su composición responde a un similar esquema que observamos en la estampa. En la zona inferior sitúa al mandatario ordenante de los suplicios, distinguido por el cetro, su disposición ecuestre y una indumentaria oriental con turbante, tanto para él como para su séquito, que visten como los turcos contemporáneos. Se trata del rey persa Saporat cumpliendo el mandato de los empe-

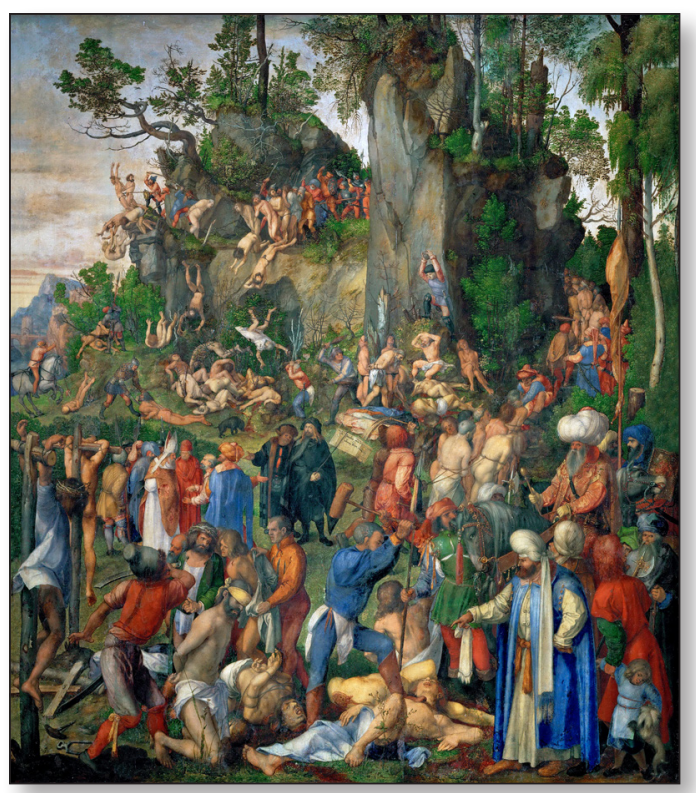

Fig. 1. Alberto Durero, Los diez mil mártires en el monte Ararat, 1508. Viena, Kunsthistoriches Museum. 
radores Adriano y Antonio. Al igual que la estampa, los reos, semidesnudos, caminan por la montaña para ser arrojados al vacío, dos de los cuales levantan sus manos como en la entalladura. Estampa y pintura destacan el ascenso de los mártires hacia la cima para ser despeñados. La fuente proviene del original griego del siglo IX atribuido a Anastasio el Bibliotecario. Destaca, en el centro de la composición, el autorretrato de Durero, que lleva en las manos una especie de cartellino en el que firmó y fechó la obra: «Este trabajo se realizó en el año 1508 por Alberto Durero, alemán».

Pero tanto el grabado como la pintura no recogen el cumplimiento de la promesa divina, es decir, la llegada en cuerpo y alma de los mártires al espacio celeste. En el mismo sentido se presenta la pintura de Tosini, en colaboración con Ridolfo Ghirlandaio, realizada hacia 1550 y conservada en el museo Florentino Salvi. El lienzo centra la composición en el diálogo de los dos emperadores que pronuncian el castigo a los reos arrodillados en la zona inferior. La batalla amanece a la derecha y, en la zona superior, se da cuenta del suplicio, la crucifixión de los mártires, pero no de la salvación celestial.

\section{DE LA PINTURA EN CARPACCIO}

Hacia el año 1515, Vittore Carpaccio compuso el tipo iconográfico sobre los diez mil mártires del monte Ararat en la pintura (tempera 307 x 205 cm., Venecia, Gallerie dell'Accademia) [fig. 2]. Había sido elaborado para un altar lateral del convento, hoy desaparecido, de san Antonio di Castello. La obra de Carpaccio tuvo su importancia, -incluso fue copiada por Tintoretto en el año 1538-, y en la actualidad se conserva solamente la parte inferior en la misma Galería de la Academia de Venecia. Sabemos que su patrocinador, Ettore Ottobon, la mandó realizar para conmemorar la victoria sobre

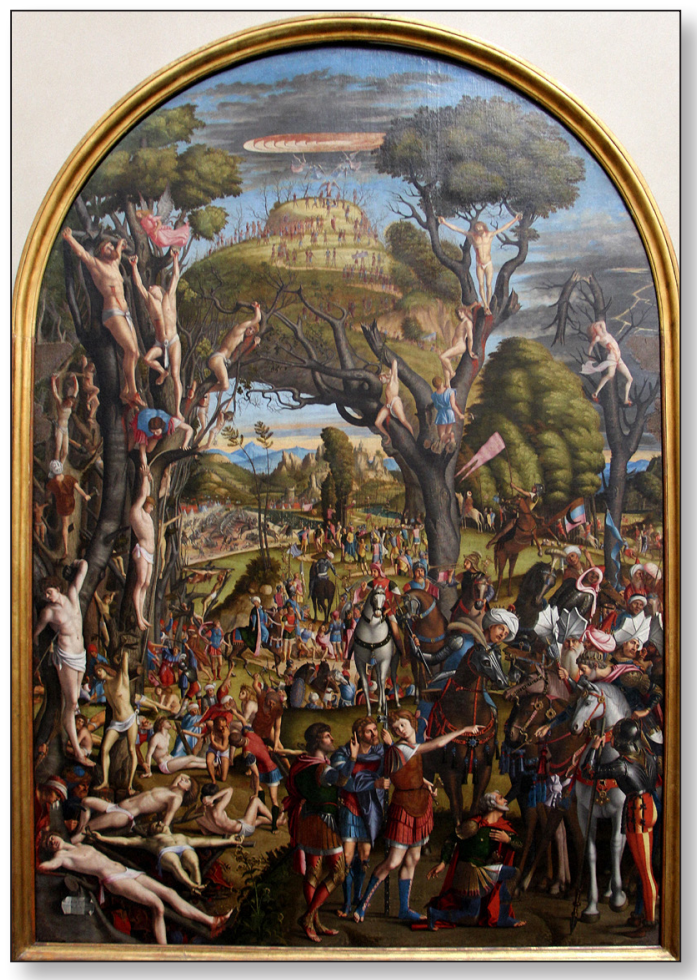

Fig 2. Vittore Carpaccio, Los diez mil mártires en el monte Ararat, 1515. Venecia, Gallerie dell'Accademia.

los turcos, donde su familia participó. La pintura, como explica Augusto Gentelli en su monografía sobre el pintor, era una denuncia a la alianza del emperador Maximiliano con el sultán otomano en detrimento de los intereses de Venecia.

Tres árboles centran la composición. Bajo ellos observamos un diálogo entre personajes sobre cabalgaduras con cuatro guerreros vestidos a la usanza romana. Uno de ellos, que muy bien responde al centurión Acacio, se arrodilla implorante. La decisión parece tomada y, a su misma altura, observamos un innumerable grupo de jóvenes semidesnudos, algunos siendo crucificados. Los troncos de los tres árboles se disponen a modo de leño para diferentes y nuevas crucifixiones en las que se hace visible tanto la llaga del costado como la corona de espinas. Todo un peregrinaje de cuerpos asciende una montaña en cuya cumbre son recibidos 


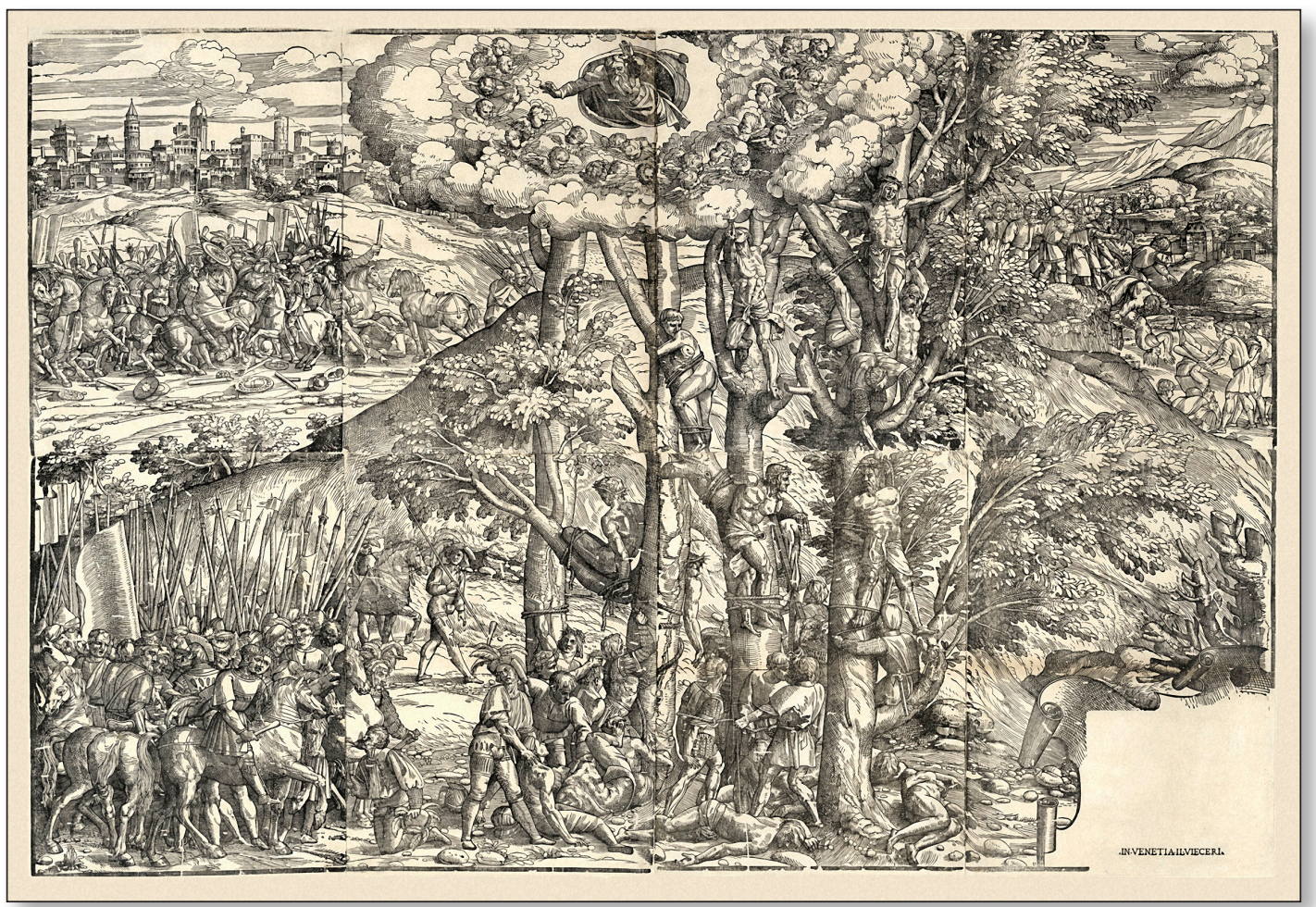

Fig. 3. Lucantonio degli Uberti, Los diez mil mártires en el monte Ararat, buril ca. 1515, impreso en el s. XVII, Yale University, Art Gallery.

por los ángeles. Estos seres espirituales los reciben y transportan hacia unos círculos concéntricos que coronan la composición, se trata de la resurrección en cuerpo y alma siguiendo la narración del martirologio para el cumplimiento de la divina promesa.

Un grabado italiano abierto por Lucantonio degli Uberti sobre invención atribuida a Tiziano y editada por Vieceri, recoge también el tema (ca. 1515, impreso en el s. XVII, Yale University, Art Gallery) [fig. 3]. La inscripción L parece responder a este grabador que elabora estampas para el citado editor. La lámina recoge la batalla y la decisión del martirio por el poder que ordena los suplicios y torturas, así lo apreciamos en los árboles donde, semidesnudos, son crucificados los mártires. Observando detenidamente la figura crucificada y coronada de espinas que se dispone en la zona superior derecha, podemos reparar en cierta identi- dad con la pintura de Carpaccio. Como en Carpaccio, la estampa recoge la promesa divina y, a través nuevamente del círculo, se presenta al Padre envuelto por la gloria de sus ángeles.

La iglesia del convento de San Antonio di Castello dispuso en la misma fecha de otra pintura de Carpaccio donde, y por encargo de su prior Francesco también de la familia Ottobon, se representó una procesión en el interior del templo que respondía a los señalados diez mil mártires de Ararat. Francesco mandó elaborar un temple sobre tabla para conformar visualmente uno de sus sueños que tuvo en el año 1511 y que se puede titular como Visión del prior Ottobon con aparición de los mártires del Monte Ararat en la iglesia de San Antonio di Castello (ca. 1515, Venecia, Gallerie dell'Accademia) [fig. 4]. Nos cuenta que se vio en la oración en la iglesia del monasterio y escuchó un gran estruendo al 


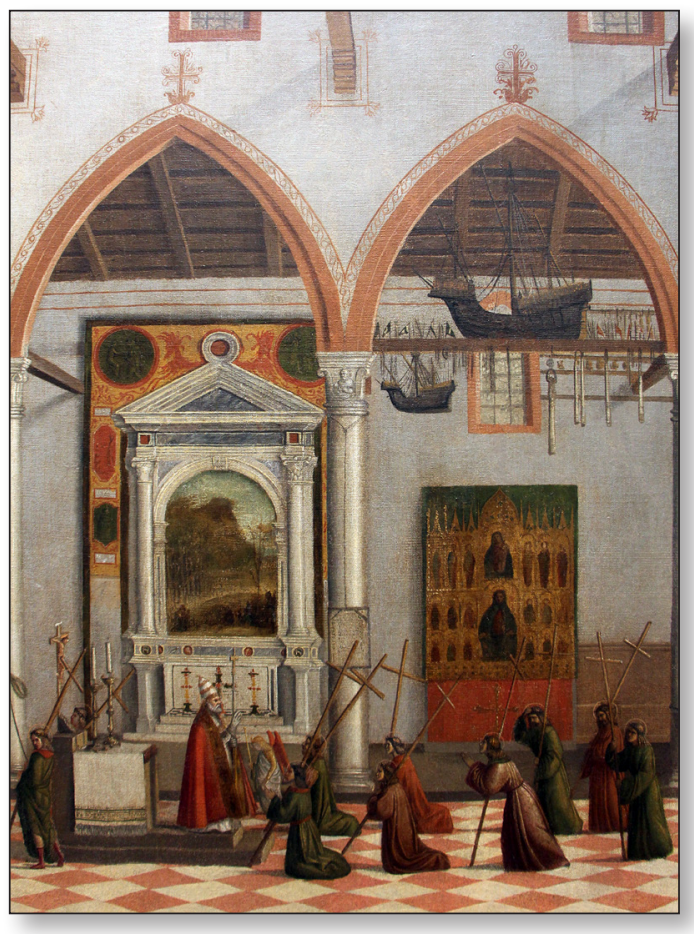

Fig. 4. Vittore Carpaccio, Visión del prior Ottobon con aparición de los mártires del Monte Ararat en la iglesia de San Antonio di Castello, ca. 1515 (det.). Venecia, Gallerie dell'Accademia.

exterior y las puertas de la iglesia se abrieron. Una multitud de hombres, portando cruces, comenzó a caminar en procesión por el pasillo. En el altar mayor se arrodillaron y fueron bendecidos por una figura a quien Ottobon identifica con San Pedro. Atravesaron, "de dos en dos, resonando con dulzura los himnos y canciones». Ottobon identificó a los peregrinos en su sueño como los diez mil mártires del monte Ararat.

\section{DANTE Y ARQUITECTURA CÓSMICA EN LA DIVINA COMEDIA}

Curiosamente, analizando el afamado poema del florentino Dante que culminara a comienzos del siglo XIV, encontramos as- pectos esenciales que nos permiten observar toda una riqueza icónica planteada por Carpaccio y que proviene de la Divina Comedia.

Sabido es que en esta cosmogonía, el poeta establece los estadios infernales a través de una estructura cónica dispuesta bajo la ciudad santa de Jerusalén. A través de la misma y de la zona superior a la inferior, se presentan los diferentes pecados en su grado de maldad, todo ello acompañado de los castigos que de cada comportamiento son merecedores. Asín Palacios ya analizó la dependencia y huella de estas manifestaciones con los Hádices musulmanes. Entre los infernales ríos y acompañado de Virgilio -que en la Eneida describe el viaje de Eneas al Hades-, se disponen los no bautizados, lujuriosos, glotones, avaros y pródigos, iracundos, herejes, violentos, fraudulentos y, finalmente, aquellos que cometieron el peor de los pecados: la traición, a quienes engulle Lucifer. Sobre el infierno se establece la puerta con el ángel guardián. Acompañado nuevamente de Virgilio, el poeta llega al Purgatorio, lugar de purificación. Si el infierno se establece a modo de montaña invertida, ahora son siete los estadios a superar que responden a la Soberbia, la Envidia, la Ira, la Pereza, la Avaricia, la Gula y la Lujuria; es decir, los siete pecados capitales definidos en tiempos del Papa Gregorio. El coronamiento, la cumbre de esta purgativa montaña, queda ocupado por el río Leteo o del olvido que sirve de entrada al Paraíso Terrenal en el que se encuentra otra fuente, el río Eunoé, de los buenos recuerdos. Es en este espacio donde Dante abandona a Virgilio y lo hace por ser pagano. Beatriz será la nueva guía, la conductora por los celestes espacios.

La estructura planetaria geocéntrica establece los diferentes estadios celestes de los que Cicerón diera cuenta en su escrito sobre el Sueño de Escipión y que se recrea en el escrito muy difundido en la época conocido como el Corpus Hermeticum, obra que influyera sin duda en el planteamiento astral que 
ofrece Ficino. Viene en primer lugar y en su proximidad a la tierra, el planeta o estrella errante: la Luna, destinado a quienes no cumplieron sus votos religiosos. Le sucede Mercurio donde reposarán quienes, por sus buenas acciones, alcanzaron gloria. Sigue Venus para las almas amorosas. El Sol Corresponde a los teólogos. Los mártires reposan en Marte. Los justos en Júpiter. Saturno acogerá los espíritus contemplativos. Sobre esta estructura se dispone el círculo de la estrellas, que es el octavo cielo, y sobre él, el noveno cielo compuesto por una estructura circular y plana como apreciamos en el tarot atribuido a Mantegna. El gran círculo dispone en su interior de otros nueve encargados de dar movimiento a todo el universo. Se trata del Primer Móvil. Finalmente, se llega al Empíreo donde una singular rosa con luz cegadora no permite contemplar a Dios. Es la expresión de la Santísima Trinidad.

Carpaccio nos presenta, como se ha señalado, estos círculos concéntricos de los que descienden los ángeles para transportar a las almas. Y lo hace porque, siguiendo a Dante (Paraíso, XXVIII), el Primer Móvil es la morada de los ángeles, los nueve anillos de los espíritus angélicos. Se recuerda con ello las nueve jerarquías de los ángeles que señalara el Pseudo Dionisio Areopagita (Jerarquía Celeste, VII, VIII y IX) y que sigue el poeta florentino: serafines, querubines, tronos, dominaciones, virtudes, potestades, principados, arcángeles y ángeles. Así lo relata:

[...] los círculos primeros / te mostraron Serafines y Querubes. [...] Aquellos otros amores que les van en torno / se llaman Tronos del divino rostro, / son los que terminan el primer ternario. [...] En esa alegría están las otras diosas: / primero Dominaciones, y luego Virtudes; / el tercer orden de las Potestades es. / Luego en los dos penúltimos júbilos / Principados y Arcángeles giran; / el último es todo de los Angélicos festejos. Todos estos órdenes hacia arriba miran, / y al inferior superan, de modo que hacia Dios / todos están siendo tirados y todos tiran.
El Primer Móvil se presenta como la gran estructura del universo que Dios mueve en su poder, con ello, todo lo creado toma vida. Así, tras dar cuenta de los nueve círculos, pone en boca de Beatriz:

\begin{abstract}
Así distante en torno al punto un cerco de fuego / giraba tan vertiginoso, que habría vencido / el movimiento que más veloz al mundo ciñe: / Y este era de otro circunscrito, / y este del tercio, y luego el tercio del cuarto, / del quinto el cuarto, y luego del sexto el quinto. / Encima sigue el séptimo tan disperso / en anchura que el correo de Juno / a contenerlo por entero sería estrecho. Así el octavo y el noveno; y cada uno I más lento se movía, conforme era su número más distante del uno. Mi dama que me veía ocupado / tan en suspenso, dijo: De aquel punto / depende el cielo y toda la natura.
\end{abstract}

Es el lugar de los ángeles, es el lugar donde los diez mil mártires resucitan en alma y cuerpo porque es el espacio donde se puede contemplar esa luz cegadora de Dios de la que dimos cuenta. Así lo relata en su canto XXX:

Como súbito relámpago que dispersa / los espíritus visivos, tal que priva / al ojo de ver más fuertes objetos, I así me circundó una luz viva, / y dejóme cegado con tal velo / su fulgor, que nada aparecía. / Siempre el amor que aquieta este cielo / con este saludo al que llega acoge / a fin de disponer a su llama la candela.

El artista, en su composición, explica con claridad, el cumplimiento de la divina promesa, la resurrección en cuerpo y alma que los ángeles trasportan al celeste espacio de las moradas espirituales, los nueve círculos, el Primer Móvil. 
